\title{
The 2012 American Heart Association/American Stroke Association International Stroke Conference
}

\author{
Frank C Barone ${ }^{* 1}$, Mateusz G Adamski ${ }^{1,2}$ \& Alison E Baird' \\ 'Neurology, SUNY Downstate Medical Center, Brooklyn, NY, USA \\ 2Neurology Jagiellonian Medical College, Krakow, Poland \\ *Author for correspondence: frank.barone@downstate.edu
}

\section{The 2012 American Heart Association/American Stroke Association International Stroke Conference}

\section{New Orleans, LA, USA, 1-3 February 2012}

The 2012 International Stroke Conference was held in New Orleans (LA, USA) between the 1st and 3rd of February. The conference maintained a focus on young investigators and career advice, including a session by the $\mathrm{NIH} / \mathrm{NINDS}$ on how to get funding, with additional special sessions and lunches devoted to stimulating young investigators. Another focus of the meeting was on translational science. The performance of a number of outstanding scientists was recognized. Here we list several highlights from the meeting in more detail.

The 2012 International Stroke Conference (i.e., the American Heart Association Stroke Council Meeting), held in New Orleans (LA, USA) between 1st February and 3rd February, was attended by 4500 researchers, practitioners and other members of the stroke community. Over 1000 posters, presentations and lectures were presented in various symposia and theme topics. The conference was preceded with a State-of-the-Art Nursing Symposium and a Pre-conference Symposium that covered novel stroke rehabilitation approaches. The conference awarded the performance of many outstanding scientists and maintained a focus on young investigators and career advice, including a session by the NIH/NINDS on how to get funding, with additional special sessions and lunches devoted to stimulating young investigators.

\section{Clinical update}

On the clinical front, results from the WARCEF trial were presented in the final presentation of the meeting by S Homma and JL Thompson (Columbia University Medical Center, NY, USA). Warfarin is often used for secondary stroke prevention in patients with low cardiac ejection fraction but this treatment approach has never been formally tested against the alternative approach of aspirin. It was reported that in patients with cardiac ejection fractions of $\leq 35 \%$ (the normal being over 50-55\%), aspirin and warfarin were equivalent for stroke prevention for up to 4 years, although after 4 years there may be cross-over between the two treatment arms. So for now, most physicians can use aspirin for secondary prevention in this patient group. It is of interest to know whether the results held up for the most severe patients with ejection fractions of $<20 \%$ (the average ejection fraction in the trial was $\sim 25 \%$ ). Lively discussions were held about the use of the novel direct thrombin inhibitors and factor Xa inhibitors for stroke prevention in patients with nonvalvular atrial fibrillation and also their use in the setting of thrombolytic therapy. The stroke community still seems divided as to the relative risks and benefits of dabigatran and rivaroxaban (relative to warfarin) and there are significant concerns over the difficulties of rapidly reversing these agents. In addition, recently the Joint Commission criteria for comprehensive stroke centers have been rolled out and can be found at the Joint Commission website [101].

The results of several recent trials that were stopped prematurely were also presented. O Benavente (University British Columbia, Canada) described results from the antiplatelet arm in the SPS3 trial. This head-to-head comparison of the combination of aspirin and clopidogrel versus aspirin alone was terminated owing to an excess mortality in the aspirin and clopidogrel group (due to noncerebral bleeding) but the antihypertensive arm continues. C Derdeyn (Washington University, St. Louis,
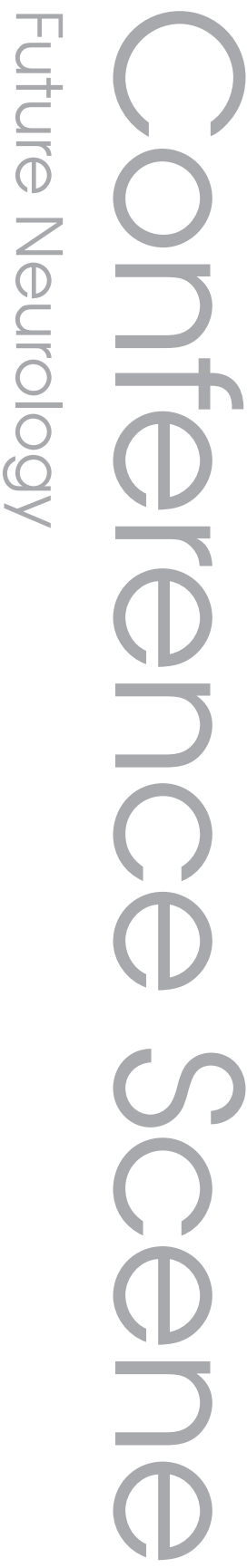

\section{Keywords}

- AHA International Stroke Conference $\approx$ basic science - cell therapy = clinical science $=$ hemorrhagic stroke - immune system in stroke - ischemic stroke = stroke clinical trials = translational medicine $=$ vascular cognitive impairment

\section{Future $\because$ Medicine part of}


MO, USA) reported on the SAMMPRIS trial and the reasons for failure in the stenting arm. In this study of patients with nondisabling stroke in the cranial arteries, superior outcomes were found for the group of patients only receiving aggressive medical therapy. This study was terminated after $60 \%$ of the planned recruitment because of the higher risks in the intervention group. Derdeyn expanded on the results of the group in which patients were treated with angioplasty and stenting in addition to medical therapy. No factors were identified that were predictive of procedure-related complications and most of the complications occurred in the first week. EB Ringelstein (University Münster, Germany) reported on the use of AX2000 for the treatment of acute ischemic stroke. Despite extensive preclinical data, this granulocyte-colony stimulating factor did not work in the clinical setting in the Phase II clinical trial and has been abandoned.

Results from a number of ongoing and recently completed clinical trials can be expected in the near future. The FAST-MAG trial has $87 \%$ of its recruitment completed. This trial of the ultra-early administration of magnesium, with approximately $70 \%$ patients suffering ischemic stroke and around 27\% with cerebral hemorrhages (and a surprisingly low rate of $3 \%$ of mimics) would, if positive, be a landmark study of neuroprotection for stroke. The MR RESCUE trial has completed its enrollment of 120 subjects. In the use of novel, clot-removal devices in acute, ischemic stroke, reperfusion using the SOLITAIRETM occluder was found to be significantly superior to that using the MERCI concentric retriever. However, these still need to be tested to determine if they improve patient outcome. These clinical findings and unanswered questions serve to address the issue of the need for more translational science. The failed clinical trials also serve to address the need for novel and high-risk lines of research.

\section{Vascular cognitive impairment}

In addition to information from clinical trials of stroke prevention and acute stroke treatment, there was significant coverage of research on cognitive impairment resulting from cerebrovascular disease. Sessions/symposiums included a particular focus on relationships to pediatric stroke and to Alzheimer's disease and on the importance of identifying models, biomarkers and neuropsychological profiles, in addition to many posters on the topic. One session that went beyond strokes and their contribution to vascular dementia, moderated by PB Gorelick (University of Illinois College of Medicine, IL, USA) and JG Merino (Stroke Center, Suburban Hospital, MD, USA) was entitled "Reversible vascular dementia". Here, present highlighted translational models, biomarkers and the importance of vascular risk factors in the development of human and animal 'dementia' (FC Barone, SUNY Downstate Medical Center, NY, USA), the importance of carotid artery disease management and surgical intervention (K Chida, Iwate Medical University, Japan), cardiac failure, low ejection fraction and reversibility of cognitive impairments, suggesting that an improvement from heart failure may also improve cognition (RM Lazar, Columbia University, NY, USA) and the inflammatory form of cerebral amyloid angiopathy that is both treatable and reversible (A Viswanathan, Harvard Medical School, MA, USA). During the concluding panel discussion, the importance of brain inflammation in developing brain pathology and symptoms, and the significant need for biomarkers in cognitive decline and dementia recovery were emphasized.

\section{Immune system \& cell therapy in stroke}

Two basic science papers on gamma-delta $\mathrm{T}$ lymphocytes in stroke injury evolution [1] and anti-inflammatory $\mathrm{T}$ regulatory lymphocytes in stroke cerebroprotection [2] have provided enthusiasm and focus for research on the immune system in stroke. This topic was discussed during several sessions and was expanded into the less-explored role of immune cell therapy, role of stroke antigens and spleen changes and bone marrow as potential treatment targets. These were addressed in a session entitled "Splenic and other immune responses to stroke" moderated by R Veltkamp (University of Heidelberg, Germany) and K Pennypacker (University South Florida FL, USA), and in many oral and poster sessions that focused on a much-repeated 'Experimental mechanisms and models' session theme). Both the innate immune system (i.e., mainly granulocytes) and the adaptive immune system (i.e., T-regulatory lymphocytes) play active roles in acute stroke pathology and brain injury. Although most results come from experimental (animal) stroke models, the initial clinical (human) data cross-validate the preclinical results and are very promising for translational work in the future. 
The spleen, which is a reservoir of red blood cells and leukocytes, is controlled by the autonomic nerve system. Both stress and stroke cause a reversible decrease in spleen mass via adrenergic neuroregulation. This is well documented, both in experimental models and human stroke. Thus, red blood cells released from the spleen under these conditions can deliver additional oxygen to stressed/ischemic tissue. Less well understood are the functions of leucocytes released under these conditions. Here, lymphocytes were a focus of significant discussion. CD3 $\mathrm{T}$ lymphocytes and natural killer (NK) lymphocytes identified in the ischemic brain of mice after stroke were found to originate from the spleen. Both spleen size and function are correlated with stroke severity/outcome in clinical settings. In addition, splenectomy improves experimental stroke outcomes. Clearly, the specificity of spleen-stroke interactions needs more detailed investigation and this is now a strong focus of translational studies and cell therapy intervention research.

Many presentations demonstrated the safety of cell-based stroke therapies in humans. The tissue distribution of administered cells is unequal across the organs, with the largest cell numbers in the spleen and brain (SI Savitz, University of Texas Medical School, Houston, TX, USA). Cells sensitized to brain antigens like myelin oligodendroglia glycoprotein (MOG) were shown to exacerbate stroke severity (PD Hurn, Oregon Health and Science University, OR, USA). Although the role of lymphocytes in the adaptive immune system in stroke is not yet understood, a possible leakage of brain antigens into the ischemic brain, producing a sterile inflammation-immune response might, explain some aspects of leukocyte function in the acute phase of stroke. These data provide important new stroke intervention opportunities and discoveries for the future.

\section{Basic \& translational science}

Multiple studies demonstrated the importance of cerebrovascular disease risk in stroke and its treatment. Several sessions (e.g., "Basic and translational neuroscience of stroke recovery and multidisciplinary clinical rehabilitation" moderated by CM Buetefisch, West Virginia University Hospital, WV, USA, and M Levin, McGill University, Montreal, Canada, and "Basic and Translational Neuroscience of Stroke Recovery" moderated by J Aronowski, University of Texas Medical School and
M Chopp, Henry Ford Hospital, Detroit, MI, USA, to name only two of many) provided updates on potential new targets/strategies for brain protection from stroke injury and restoration of function post-stroke. Mechanisms included, among others, the upregulating of endogenous protection mechanisms, the role of synaptic remodeling in brain recovery and cell-based intervention approaches. Numerous clinical and preclinical studies demonstrated the importance of imaging the white matter injury involved in long-term cognitive impairment from cerebrovascular disease. The important need for other biological markers of cerebral microvascular disease and blood flow deregulation, neurovascular dysfunction, oxidative stress and inflammation in brain injury and long-term disruption of sensory, motor and cognitive functions were recurring themes that we can expect to impact upon both future discoveries and new research directions.

\section{Awards}

Several prestigious awards were announced during the plenary sessions. The awards recognized the outstanding contributions of these individuals to the field of stroke and stroke research. WD Heiss (Max Planck Institute and University of Cologne, Germany) received the David G Sherman Lecture Award for lifetime contributions to the investigation, management, mentorship and community service in the basic or clinical stroke field. His award lecture was entitled "The role of positron emission tomography for translational research in stroke". Heiss is recognized for delineating blood flow thresholds on tissue vulnerability and the transition from reversibility to tissue death or recovery. His groundbreaking PET and MRI studies developed threshold maps for tissue viability and allowed the study of the penumbra (i.e., the ischemic stroke brain areas that can be salvaged by intervention).

GJ del Zoppo (Washington University School of Medicine, St. Louis, MO, USA) received the Thomas Willis Award for basic science investigation in stroke. He delivered the Award Lecture entitled "Toward the Neurovascular Unit: A Journey in Clinical Translation". del Zoppo is recognized for his clinical and basic expertise in stroke thrombosis and cerebral vascular biology and his fundamental discoveries in understanding brain vessel thrombosis and microvessel responses to reduced blood flow that contribute to stroke injury. He was a key leader in three of the first 
multicenter, international prospective trials of clot busters for ischemic stroke (i.e., trials of acute intra-arterial and intravenous plasminogen activator). He and his group formulated today's conceptual framework of the 'neurovascular unit'.

JL Saver (UCLA Geffen School of Medicine and Stroke Center, CA, USA) received the William Feinberg Award for Excellence in Clinical Stroke. Saver has been the principal investigator in numerous trials spanning a spectrum of stroke care. He gave the award lecture entitled "Treatment swift and treatment sure: prehospital neuroprotection and highly effective endovascular recanalization therapy for acute ischemic stroke". Saver has made many pioneering contributions, including advancing the prehospital identification and treatment of stroke, modernizing the definition of transient ischemic attack, developing methods to analyze the whole range of poststroke deficits in clinical trials, showing that rapid restoration of blood flow can reverse acute stroke brain injury and developing clot retrieval devices to reopen blocked cerebral arteries in acute stroke.

The other awards recognized research presented by young investigators. A Towfighi (University of California at Los Angeles and Rancho Los Amigos National Rehabilitation Center, Los Angeles, CA, USA) received the Robert G. Siekert New Investigator in Stroke Award. Her abstract and presentation was entitled "High Framingham Cardiovascular Risk Scores are of Prognostic Value in Recent Ischemic Stroke Patients without known Coronary Heart Disease". C Lin (Duke-National University of Singapore, Republic of Singapore and Duke Clinical Research Institute, Durham, NC, USA) received the Stroke Care in Emergency Medicine Award. Her abstract and presentation was entitled "Pre-notification by Emergency Medical Services is Associated with More Timely Evaluation and Treatment of Acute Ischemic Stroke". Finally, A Sarraj (University of Texas, Houston, TX, USA) received the Mordecai Y.T. Globus New Investigator Award. Her abstract and presentation was entitled "Optimizing
Prediction Scores for Poor Outcome After Intraarterial Therapy for Anterior Circulation Acute Ischemic Stroke".

\section{Conclusion}

The 2012 International Stroke Conference provided updates on ongoing trials in addition to overviews and focused oral and poster sessions on new and emerging science. A key theme throughout the meeting was 'translational science' as related to integrating basic and clinical science research and to extending knowledge on mechanisms and biomarkers in cerebrovascular disease/stroke. Many outstanding researchers, from young to old and with focus from the very basic to clinical disease science were recognized. As in the past, this years American Heart Association/American Stroke Association International Stroke Conference meeting was exciting and informative from beginning to end.

\section{Financial \& competing interests disclosure}

The authors have no relevant affiliations or financial involvement with any organization or entity with a financial interest in or financial conflict with the subject matter or materials discussed in the manuscript. This includes employment, consultancies, honoraria, stock ownership or options, expert testimony, grants or patents received or pending, or royalties.

No writing assistance was utilized in the production of this manuscript.

\section{References}

1. Shichita T, Sugiyama Y, Ooboshi $\mathrm{H}$ et al. Pivotal role of cerebral interleukin-17-producing $\gamma \delta \mathrm{T}$ cells in the delayed phase of ischemic brain injury. Nat. Med. 15(8), 946-950 (2009).

2. Liesz A, Suri-Payer E, Veltkamp C et al. Regulatory $\mathrm{T}$ cells are key cerebroprotective immunomodulators in acute experimental stroke. Nat. Med. 15(2), 192-199 (2009).

\section{Website}

101. Advanced Certification Comprehensive Stroke Centers.

www.jointcommission.org/certification/advanced_ certification_comprehensive_stroke_centers.aspx 